\title{
REVIEW
}

\section{Role of surgery in the management of the adult patient with coarctation of the aorta}

\section{Ramnarine}

Postgrad Med J 2005;81:243-247. doi: 10.1136/pgmj.2004.024588

Adult patients with coarctation of the aorta have a range of clinical presentations. These include the presence of additional cardiovascular anomalies (predominantly aortic valve abnormality) and presentation with complications after coarctation repair in childhood (such as recurrent coarctation or aneurysm formation). Developments in endovascular technology over the past decade may potentially reduce the morbidity from open surgical repair. However, some cases are unsuitable for endovascular repair and open surgical techniques continue to play a part in the management of these patients. The number of adult patients with coarctation is very small and a trial comparing surgical and endovascular repair techniques would be difficult to arrange. A multidisciplinary team (consisting of cardiothoracic surgeon, interventional radiologist, cardiologist, and anaesthetist) is best equipped to manage the unique and complex problems that affect these patients. The current surgical options and factors governing the choice of approach are reviewed.

Correspondence to: Mr I Ramnarine, Department of Human Anatomy and Cell Biology, University of Liverpool, Liverpool L69 3GE, UK. iramn@liverpool.ac.uk

Submitted 25 May 2004 Accepted 25 August 2004

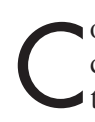
oarctation of the aorta ( $\mathrm{CoA})$ describes the congenital narrowing of any region from the distal aortic arch to the abdominal aortic bifurcation. There has been a considerable body of work covering repair in childhood. However, the adult patient with CoA presents with different problems. Open surgical repair is associated with morbidity and mortality but is often the only option. Recent developments in endovascular stent technology have extended the treatment options available and seem cost effective. ${ }^{1}$ Results have generally been excellent despite the small risk of aortic rupture. ${ }^{2}$ The number of cases is still small and there have been no controlled trials testing the relative benefits of endovascular over open surgical repair. Most cases of isolated CoA are suitable for an initial attempt at endovascular repair. However, complex cases such as those with additional cardiac abnormality, with aneurysms, or stenosis after previous CoA repair are better managed by a multidisciplinary team consisting of cardiothoracic surgeon, interventional radiologist, cardiologist, and anaesthetist.

The current trends in the management of the adult patient with CoA and the factors that govern the approach taken for the patient are reviewed.

\section{ANATOMY}

The common site of aortic narrowing is adjacent to the ductus arteriosus and is thought to be attributable to overgrowth of ductal tissue into the aorta. The crude classification into adult and infantile types does not accurately reflect the clinical presentations because many "infantiletype" have presented in adulthood. In the preductal (infantile) type of CoA the narrowing lies proximal to the ductus arteriosus. Distal perfusion is dependent on blood flow through the ductus arteriosus and acute distal circulatory shutdown follows closure of the duct. The postductal (adult) type of CoA often occurs at or just distal to the ductus arteriosus and the obstruction is seldom complete (see fig 1). Classification of the lesion into discrete narrowing, isthmic hypoplasia (the narrowing is $<75 \%$ of the transverse aortic diameter), and tubular or diffuse stenosis (the narrowing is $>10 \mathrm{~mm}$ ) is important for endovascular intervention. ${ }^{3}$ Collateral flow from several sites (around the scapula and thorax) is responsible for distal perfusion. Patients are often completely asymptomatic well into the fifth decade. About $10 \%$ of adult CoA occurs in the distal descending or abdominal aorta. ${ }^{4}$ The interrupted aortic arch represents a part of the spectrum of CoA and is exceedingly rare.

CoA is associated with a high incidence of other congenital abnormalities, predominantly of the left ventricular outflow tract. A bicuspid aortic valve is present in about a third of patients and other common anomalies include subvalvar aortic stenosis and mitral valve abnormal$\operatorname{ity}(3 \%) .^{5}$

\section{CLINICAL FEATURES}

Most adult patients with CoA are completely asymptomatic and the diagnosis is only made when investigated for the cause of hypertension. If left untreated, death occurs most often in the fifth decade. ${ }^{6}$ Other features include lower limb claudication, headaches, vomiting, and feeling dizzy. Occasionally patients present in cardiac failure or with infective endocarditis, aortic dissection, or cerebrovascular accidents. ${ }^{7}$ Typically the femoral pulses are absent or there may be a delay between the radial and femoral pulses. The presence of well developed collateral arteries around the scapulae can often be appreciated by palpation or auscultation. A systolic murmur may be heard in the axilla and back. The electrocardiogram almost always shows evidence of left ventricular strain in keeping with hypertension. The chest radiograph may show cardiomegaly and rib notching. 


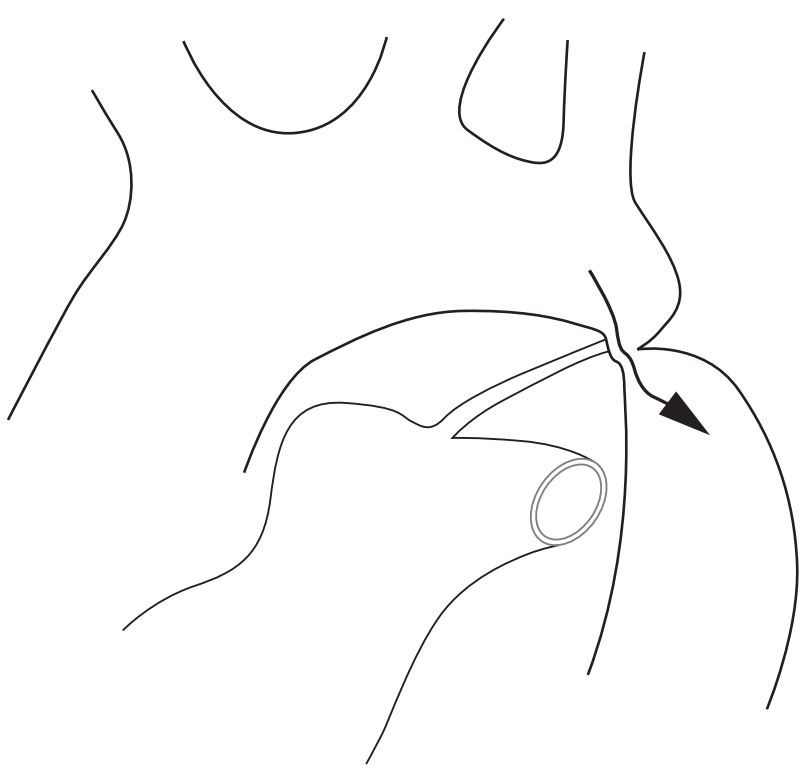

Figure 1 Postductal or adult type coarctation of the aorta. The ductus arteriosus is closed off and now forms the ligamentum arteriosus. The stenosis is adjacent to the ligamentum and some blood flows through the lesion.

Patients who have had previous coarctation repair surgery in childhood are often monitored, but some may present again with hypertension and the symptoms listed above.

\section{IMAGING}

All patients should have a chest radiograph to show rib notching and to assess for cardiomegaly. Other abnormal cardiac silhouettes may raise the suspicion of the presence of other cardiac anomalies.

Echocardiography is essential for the assessment of aortic, mitral, and left ventricular structure and function. This information is vital because the presence of other cardiac anomalies may require additional corrective procedures to be performed and may also have an impact on the postoperative course. The suprasternal probe position provides views of the CoA and the gradient across the stenosis can be calculated using Doppler ultrasound.

The gold standard imaging technique has been aortography. In addition to providing accurate images, the pressure gradient across the coarctation can be assessed and a guide wire may be placed across the lesion. If the lesion cannot be crossed separate catheters above and below could provide the same information. The placement of endovascular stents and balloon dilatations both require that a guide wire is passed across the lesion. Therefore, if the lesion cannot be crossed (that is, the coarctation is complete) surgery would be the treatment of choice. Fortunately, this rarely occurs.

Aortography does not provide much information about the tissue surrounding the aorta. Computed tomography and magnetic resonance imaging can provide this information, especially when combined with three dimensional reconstruction software. Unfortunately, no pressure information can be obtained. Almost all patients require multiple investigations to be performed.

\section{ANAESTHESIA}

The anaesthetist is a key member of the multidisciplinary team managing these patients during intervention. Whether or not additional cardiac lesions are present, a considerable amount of cardiac strain patient may be experienced (such as during balloon dilatation or surgery). It is essential to minimise this. Temperature, invasive arterial, and cardiac monitoring supplement routine intraoperative monitoring (ECG, oximetry, capnography, etc). Often two arterial transducers (proximal and distal to the lesion) are necessary to monitor adequate upper and lower body perfusion. Central venous catheters and cardiac output catheters are essential for difficult cases with additional cardiac anomalies. Extremely complicated cases may also require spinal cerebrospinal fluid pressure monitoring possibly combined with cerebrospinal fluid drainage.

\section{CORRECTION OF DEFECT}

Survival is improved by surgical correction of $\mathrm{CoA}^{8}$ and the disease process is modified. The objective of repair is to permit proximal blood flow to pass distally without obstruction. This can be achieved by either widening the narrowed region or by creating an alternate path for blood flow. Surgical or endovascular techniques can be used.

\section{ENDOVASCULAR REPAIR}

A balloon is placed across the CoA and distended to relieve the stenosis. Balloon aortoplasty was initially introduced in the newborn, ${ }^{9}$ but has extended to include adults. Some debate exists over whether a stent should be placed as well, and the choice of stent material. ${ }^{3}$ There is minimal risk of morbidity, but if aortic tearing and bleeding does occur a stent may be used to limit this. Nevertheless, surgical cover should be available.

\section{SURGERY}

Historically surgery has been the treatment of choice for CoA repair. Several factors govern the choice of surgical approach and a variety of techniques have been used in the past. Table 1 describes the most common approaches.

\section{Incision}

Coarctation repair is performed through a left thoracotomy or through a median sternotomy (slightly more difficult). Left thoracotomy through the third or fourth rib bed provides adequate exposure. Numerous large arteries within the muscles and between the ribs must be divided. These collateral vessels tend to be large, friable, and thin walled because of the increased blood pressure and flow. They often quickly retract and should be transfixed and ligated as soon as they are identified or secondary haemorrhage may result.

A median sternotomy is less likely to cause bleeding problems as no muscles are cut. However, the internal mammary arteries are much larger than normal and should be carefully avoided. Combination procedures (such as valve or coronary artery surgery) are performed through this incision.

\section{Isolated coarctation}

The classic approach via left thoracotomy is most often used for isolated coarctation repair. The surgical approach and repair technique is governed by the anatomy of the lesion.

\section{End to end anastomosis}

Crafoord and Nylin ${ }^{10}$ initially introduced this approach in 1945. It is only occasionally used today providing there is sufficient normal tissue present (after resection of the lesion) to complete the repair without tension.

\section{Prosthetic interposition tube grafts}

The most commonly used technique having largely replaced the end to end anastomosis. Careful dissection is used to show the relevant anatomy and to avoid left recurrent laryngeal nerve injury. Vascular sloops or tapes are passed around the left subclavian artery, the aorta both proximal, and distal to the coarctation. The ligamentum arteriosum is 
Table 1 Surgical approaches to the management of coarctation of the aorta

\begin{tabular}{lll}
\hline Primary disease & Complex cardiac lesions & Reoperation after previous repair \\
\hline Tube interposition graft & Staged incisions & Hypothermic circulatory arrest \\
End to end repair & Combined incisions & Extra-anatomical bypass \\
Subclavial flap & Extra-anatomical bypass & Endovascular stent \\
Prosthetic patch & Endovascular stent & Balloon aortoplasty \\
Homograft & Balloon aortoplasty & \\
Endovascular stent & Combined endovascular and surgical \\
Balloon aortoplasty & & \\
\hline
\end{tabular}

transfixed and divided. Systemic intravenous heparin is given. Cardiopulmonary bypass is started if appropriate (below). Vascular clamps are applied proximal and distal to the coarctation, the narrowed segment is resected, and a prosthetic tube is interposed before de-airing and clamp release.

\section{Subclavian flap repair}

The left subclavian artery is ligated at thoracotomy. The coarctation is opened and the subclavian is used as a patch to widen the narrowed area. The arterial patch has significant growth potential. Good long term results from using this technique in childhood have been reported. It is not recommended for the adult because of the risk of upper limb ischaemia despite the presence of collateral blood supply to the left upper limb.

\section{Prosthetic patch repair}

A variety of vascular materials have been used. This entails clamping the relevant segment of aorta, opening the narrowed segment (usually longitudinally), and using a prosthetic patch to increase the luminal diameter. This technique is currently not in common use because the association with late aneurysm formation when used in childhood coarctation repairs. ${ }^{11} 12$

\section{Cardiopulmonary bypass}

Cardiopulmonary bypass or more appropriately left heart bypass must be available during surgery. This provides an essential recovery strategy in difficult cases or when complications occur. Monitoring femoral arterial pressure ensures that distal perfusion is satisfactory. During surgical repair a trial of clamping across the aorta distal to the coarctation is attempted and should the distal pressure decrease significantly, this suggests that the flow through the narrowed segment of aorta was essential to distal perfusion. Such cases should have been identified from angiography and would usually have been suitable for angioplasty. These patients should have bypass established to maintain distal perfusion. Left heart bypass can be established with left atrial drainage pumped into the femoral artery. Single lung ventilation must be continued. This permits oxygenated blood to be pumped into the peripheries. Vascular shunts have been used in the past but are difficult to fit into the operating field and bypass pumps provide much better flows. Normothermia should be maintained because cooling can cause ventricular fibrillation, especially with the hypertrophic ventricle.

An absence of a pressure decrease on clamping would suggest that the collateral flow maintains the distal perfusion and the operation can proceed without the need for to bypass.

\section{Hypothermic circulatory arrest}

Patients with previous coarctation repairs who require intervention for re-stenosis or aneurysms often have adhesions that make dissection difficult and very dangerous. Cardiopulmonary bypass can be used to bring the core body temperature down to $15^{\circ} \mathrm{C}$ and this can permit the circulation to be stopped for up to an hour. Selective carotid artery cannulation can facilitate cerebral perfusion while the rest of the circulation is stopped. Hypothermic circulatory arrest permits the circulation to be stopped while the dissection is performed, thus minimising the risk of exsanguinating haemorrhage. The dissection can be carried out and anastomoses performed before restoring cardiopulmonary bypass without mortality or significant morbidity. ${ }^{13}$

\section{Extra-anatomical correction ${ }^{14} 15$}

A useful surgical technique has been to channel aortic blood via a prosthetic conduit through a different route. This is accomplished through a sternotomy and cardiopulmonary may be used. The conduit is anastomosed to the ascending aorta then the heart is lifted and the posterior pericardium is opened to allow anastomosis to the descending thoracic aorta distal to the coarctation (see fig 2).

This technique can be used for the interrupted aortic arch and in combined procedures such as with valve ${ }^{16}$ and coronary artery surgery. ${ }^{17}$ Adhesions from previous surgery

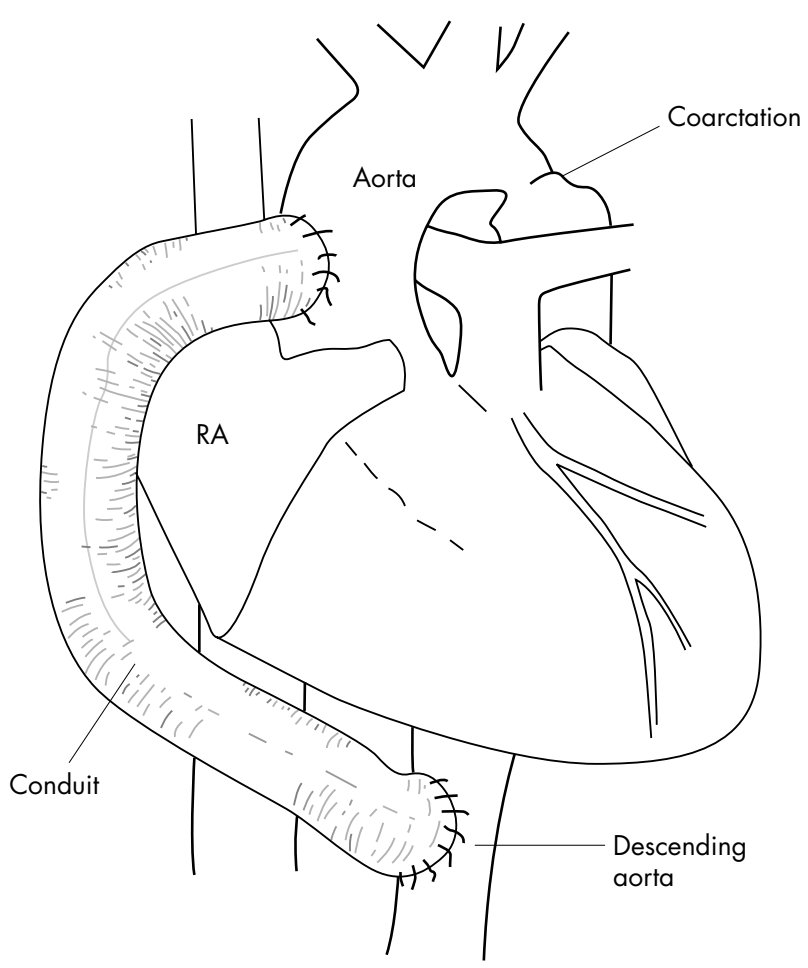

Figure 2 An extra-anatomical bypass procedure. The conduit is anastomosed from the ascending aorta and to the descending aorta "bypassing" the coarctation segment. This can be performed by median sternotomy, using cardiopulmonary bypass, and in conjunction with other cardiac surgical procedures (such as valvular or coronary artery surgery). 
may make a left thoracotomy approach difficult and dangerous and extra-anatomical correction facilitates blood through an alternate route without disturbing the adhesions.

\section{ADDITIONAL CARDIAC LESIONS}

Common additional cardiac problems that may require intervention include coronary artery disease and the repair or replacement of the aortic or mitral valve.

\section{Coronary artery disease}

Reifenstein et $a l^{18}$ were the first to note coronary artery disease in coarctation patients and acute myocardial infarction has been reported as the cause of death in about two thirds of patients with previous CoA repair. ${ }^{19}$ The adult patient with CoA is often hypertensive and at risk of developing coronary artery disease. A careful history should identify at risk patients and coronary angiography is performed at the same time as aortography, if indicated. Any attempts at CoA repair in these patients would be disastrous without prior or simultaneous coronary revascularisation. The internal mammary arteries are often increased in size and are unsuitable for use as conduit for revascularisation. ${ }^{20}$ It is possible to use a left thoracotomy to perform CoA repair and off pump coronary artery surgery simultaneously.

\section{Aortic valve disease}

Between $20 \%^{21}$ and $85 \%^{22}$ of all patients with coarctation have congenitally bicuspid aortic valves. A proper assessment of the valve including cardiac catheterisation and echocardiography should be undertaken. If the aortic abnormality is not a significant cause of haemodynamic abnormality the coarctation can be managed first and the aortic abnormality followed up. Combined aortic valve replacement and standard coarctation repair can be performed via median sternotomy ${ }^{16}$ or by using extra-anatomical bypass. ${ }^{23}$ Other options include a two staged operation (sternotomy for aortic valve replacement and thoracotomy for CoA repair), endovascular repair of the CoA followed closely by aortic valve surgery $^{24}$ or CoA repair and surveillance with aortic valve replacement when indicated.

\section{COMBINED PROCEDURES}

Endovascular devices are deployed through peripheral arteries and fluoroscopy used to confirm accurate placement. Complex anatomical variations may require endovascular devices to be deployed directly into the aorta. Both surgeon and interventional radiologist must work closely to ensure accurate placement.

\section{COMPLICATIONS OF SURGERY (TABLE 2)}

A better understanding of the difficulties and improvements in perioperative care has resulted in better surgical results. ${ }^{7}$

Table 2 Postoperative complications of coarctation of the aorta repair

\begin{tabular}{ll}
\hline Early complications & Late complications \\
\hline Hypertension & Hypertension \\
Mesenteric adenitis & Aortic disease \\
Haemorrhage & Restenosis \\
Recurrent laryngeal nerve injury & Dissection \\
hoarseness & Aneurysm formation \\
Spinal cord injury & Aortic rupture \\
paraplegia & Infective endocarditis \\
Arrhythmias & Cardiac failure \\
& Aortic valve disease \\
\hline
\end{tabular}

\section{Early}

Most patients with CoA also have hypertensive cardiac disease. The bulky ventricle is at risk of developing ischaemia and fibrillation when the afterload is increased (when the endovascular balloon is inflated or the aorta is clamped). Pharmacotherapy and cardiopulmonary bypass can be used to offload the heart and reduce cardiac stress, but vigilant monitoring is essential.

Postoperative haemorrhage is not uncommon, careful haemostasis during thoracotomy, and rigorous blood pressure control throughout are essential.

Recurrent laryngeal nerve injury can occur, especially in difficult cases such as re-operations where the anatomy is difficult to delineate. Often this requires no intervention and vocal chord movement gradually returns. Intervention is only necessary if aspiration or asphyxia occur.

If spinal perfusion is impaired paraplegia may occur. Monitoring of upper and lower body arterial pressures and the spinal cerebrospinal fluid pressures has minimised this risk. Cerebrospinal pressure monitoring is only necessary for complicated cases and often a small volume of fluid is drawn off to improve the spinal perfusion. The use of partial or left heart bypass to maintain the lower body arterial pressure can prevent paraplegia.

Post-coarctation syndrome relates to "mesenteric adenitis" that results from the increase in blood pressure to the gut after coarctation repair. Nausea, vomiting, abdominal pain, and ileus are common. Moss and $\mathrm{Ho}^{25}$ showed that blood pressure control significantly reduced the incidence of this problem and the need for laparotomy. Control of the blood pressure and fluid resuscitation would resolve most cases.

Hypertension can be both an early and late problem after coarctation repair. The use of glycerol trinitrate and sodium nitroprusside in the early postoperative period followed by oral antihypertensives before discharge is recommended. The incidence of hypertension is reduced in the long term, but exercise induced hypertension seems to have been largely unrecognised..$^{26}$

\section{Late}

Haemorrhage after hospital discharge is uncommon, but can be serious. CoA repair does reduce the incidence of hypertension but every effort should be made to control the blood pressure ${ }^{19}$ and antihypertensive drugs are often needed.

"Re-coarctation" is a misnomer. After surgical repair in childhood, the tissue around the repair does not grow relative to the rest of the patient and restenosis results. Tissue overgrowth at the anastomosis site is another cause of restenosis. Pressure gradient assessment is necessary. Balloon dilatation may provide better results than for the treatment of primary CoA. Adhesions after the previous operation make dissection in this area difficult; however, this tissue is stronger than the native aorta and responds better to dilatation. Effective surgical strategies include either direct surgical repair under hypothermic circulatory arrest or use of an extra-anatomical bypass.

Aneurysms can form at the site of previous coarctation repair $^{27}$ and can easily rupture, bleed, or lead to paraplegia. This was more common with prosthetic patch repairs. Endovascular grafts can be placed across the aneurysm if there is a suitable region of healthy aorta proximal to the aneurysm for the graft to be anchored. ${ }^{28}$

Aortic dissection and rupture can be catastrophic and are (thankfully) rare. They are very difficult to manage.

\section{CONCLUSION}

Surgery still has an important part to play in the management of the adult patient with CoA despite the recent advances of endovascular technology. Clinical trials for these 
patients are difficult to carry out because of the small patient numbers and the variety of complicating factors. The multidisciplinary team can optimise the management of these patients and combined procedures may further improve results.

Funding: none.

Conflicts of interest: none declared.

\section{REFERENCES}

1 George JC, Shim D, Bucuvalas JC, et al. Cost-effectiveness of coarctation repair strategies: endovascular stenting versus surgery. Pediatr Cardiol 2003;24:544-7.

2 Mullen MJ. Coarctation of the aorta in adults: do we need surgeons? Heart 2003;89:3-5.

3 Zabal C, Attie F, Rosas M, et al. The adult patient with native coarctation of the aorta: balloon angioplasty or primary stenting? Heart 2003;89:77-83.

4 Cherif A, Chouach H, Belhani A, et al. [Coarctation of the abdominal aorta: apropos of 4 cases with a review of the literature]. Tunis Med 1988;66:759-63.

5 Bouhour JB, Lefevre M, Nicolas G, et al. [Study of the association between aortic coarctation and congenital mitral insufficiency (excluding atrioventricular canals)]. Arch Mal Coeur Vaiss 1977:70:337-45.

6 Campbell M. Natural history of coarctation of the aorta. Br Heart J 1970;32:633-40

7 Jenkins NP, Ward C. Coarctation of the aorta: natural history and outcome after surgical treatment. Q J Med 1999;92:365-71.

8 Cheitlin MD. Coarctation of the aorta. Med Clin North Am 1977;61:655-73.

9 Singer MI, Rowen M, Dorsey TJ. Transluminal aortic balloon angioplasty for coarctation of the aorta in the newborn. Am Heart J 1982;103:131-2.

10 Crafoord C, Nylin G. Congenital coarctation of the aorta and its surgical treatment. J Thorac Surg 1945;14:347-61.

11 Parikh SR, Hurwitz RA, Hubbard JE, et al. Preoperative and postoperative "aneurysm" associated with coarctation of the aorta. J Am Coll Cardiol 1991; 17:1367-72.

12 Rheuban KS, Carpenter MA, Jedeikin R, et al. Aortic aneurysm after patch aortoplasty for coarctation in childhood. Am J Cardiol 1985;55:612.

13 Gudbjartsson T, Mathur M, Mihaljevic T, et al. Hypothermic circulatory arrest for the surgical treatment of complicated adult coarctation of the aorta. J Am Coll Cardiol 2003;41:849-51.
14 Heinemann MK, Ziemer G, Wahlers $T$, et al. Extraanatomic thoracic aortic bypass grafts: indications, techniques, and results. Eur J Cardiothorac Surg 1997; 1 1:169-75.

15 Almeida de Oliveira S, Lisboa LA, Dallan LA, et al. Extraanatomic aortic bypass for repair of aortic arch coarctation via sternotomy: midterm clinical and magnetic resonance imaging results. Ann Thorac Surg 2003;76:1962-6

16 Vijayanagar R, Natarajan P, Eckstein PF, et al. Aortic valvular insufficiency and postductal aortic coarctation in the adult. Combined surgical management through median sternotomy: a new surgical approach. J Thorac Cardiovasc Surg 1980;79:266-8

17 Bartoccioni S, Giombolini C, Fiaschini P, et al. Aortic coarctation, aortic valvular stenosis, and coronary artery disease: combined one-stage surgical therapy operation. J Card Surg 1995; 10:594-6.

18 Reifenstein GH, Levine SA, Gross RA. Coarctation of the aorta: a review of 104 autopsied cases of the 'adult-type', 2 years of age or older. Am Heart J 1947;33:146-68.

19 Lawrie GM, DeBakey ME, Morris GC Jr, et al. Late repair of coarctation of the descending thoracic aorta in 190 patients. Results up to 30 years after operation. Arch Surg 1981;116:1557-60.

20 Dunst KM, Zelger BG, Huemer GM. Severe atherosclerosis in the internal mammary artery after aortic coarctation. Eur J Cardiothorac Surg 2004;25:892-3.

21 Stewart $A B$, Ahmed R, Travill CM, et al. Coarctation of the aorta life and health 20-44 years after surgical repair. Br Heart J 1993;69:65-70.

22 Presbitero $P$, Demarie $D$, Villani $M$, et al. Long term results (15-30 years) of surgical repair of aortic coarctation. Br Heart J 1987;57:462-7.

23 Sun LZ, Luo XJ, Liu YM. Single-stage treatment of aortic coarctation and aortic valve disease. Asian Cardiovasc Thorac Ann 2003;11:208-12.

24 Magee AG, Blauth Cl, Qureshi SA. Interventional and surgical management of aortic stenosis and coarctation. Ann Thorac Surg 2001;71:713-15.

25 Ho EC, Moss AJ. The syndrome of "mesenteric arteritis" following surgical repair of aortic coarctation. Report of nine cases and review of the literature. Pediatrics 1972;49:40-5.

26 Ozyazicioglu A, Ates A, Yekeler I, et al. Repair of coarctation of the aorta in adults and hypertension. Cardiovasc Surg 2003;1 1:353-7.

27 Knyshov GV, Sitar LL, Glagola MD, et al. Aortic aneurysms at the site of the repair of coarctation of the aorta: a review of 48 patients. Ann Thorac Surg 1996:61:935-9.

28 Ince $\mathbf{H}$, Petzsch $M$, Rehders $T$, et al. Percutaneous endovascular repair of aneurysm after previous coarctation surgery. Circulation 2003;108:2967-70. 